\title{
Weight-Based Insulin Dosing for Acute Hyperkalemia Results in Less Hypoglycemia
}

\author{
Dauria T. Wheeler, PharmD1, Stephen J. Schafers, PharmD, BCPS², Tim A. Horwedel, PharmD, BCPS², \\ Eli N. Deal, PharmD, BCPS ${ }^{2 *}$, Garry S. Tobin, MD³
}

${ }^{1}$ St. Louis College of Pharmacy, St. Louis, Missouri; ${ }^{2}$ Department of Pharmacy, Barnes-Jewish Hospital, St. Louis, Missouri; ${ }^{3}$ Diabetes Center, Washington University in St. Louis Medical School, St. Louis, Missouri.

Hyperkalemia treatment with intravenous insulin has been associated with hypoglycemia. This single-center, retrospective study compared the effects on hypoglycemia between weight-based insulin dosing $(0.1 \mathrm{U} / \mathrm{kg}$ of body weight up to a maximum of $10 \mathrm{U}$ ) compared to standard flat doses of $10 \mathrm{U}$ among patients weighing less than $95 \mathrm{~kg}$. Of the 132 charts randomly selected for review, hypoglycemic events (blood glucose $<70 \mathrm{mg} / \mathrm{dL}$ ) were reduced from $27.3 \%$ in the $10-\mathrm{U}$ group to $12.1 \%$ in the weight-based group $(P=0.05)$. The number of affected patients was reduced with $19.7 \%$ in the $10-U$ group and $10.6 \%$ in the weight-based group $(P=0.22)$. The potassium-lowering effects of these 2 strategies were similar between groups. Female patients and those with baseline glucose values $<140 \mathrm{mg} / \mathrm{dL}$ were at increased risk for hypoglycemia. Weight-based insulin dosing $(0.1 \mathrm{U} / \mathrm{kg})$ for acute hyperkalemia therapy resulted in less hypoglycemia without impacting potassium lowering. Journal of Hospital Medicine 2016;11:355-357. (c) 2016 Society of Hospital Medicine
Hyperkalemia occurs in as many as $10 \%$ of all hospitalized patients, ${ }^{1}$ leading to potentially fatal arrhythmias or cardiac arrest that results from ionic imbalance within the resting membrane potential of myocardial tissue. ${ }^{2}$ Acute instances may be stabilized with insulin to stimulate intracellular uptake of potassium, but this increases the risk of hypoglycemia. ${ }^{2}$ Centers for Medicare and Medicaid Services quality measures require hospitals to minimize hypoglycemic events, particularly serious events with blood glucose (BG) $<40 \mathrm{mg} / \mathrm{dL}^{3}$ due to an association with an increase in mortality in the hospital setting. ${ }^{4}$ Previous research at our tertiary care hospital found that $8.7 \%$ of patients had suffered a hypoglycemic event following insulin administration pursuant to acute hyperkalemia treatment, and that patients with a lower body weight are at increased risk of hypoglycemia, particularly severe hypoglycemia $(\mathrm{BG}<40 \mathrm{mg} / \mathrm{dL})$. $^{5}$ Increasing the total dose of dextrose provided around the time of insulin administration is suggested to reduce this concern. ${ }^{5}$

Patients at our institution receive $50 \mathrm{~g}$ of dextrose in conjunction with intravenous (IV) insulin for hyperkalemia treatment. To further reduce the potential for hypoglycemia, our institution amended the acute hyperkalemia order set to provide prescribers an alter-

\footnotetext{
*Address for correspondence and reprint requests: Eli N. Deal, PharmD, Department of Pharmacy, Barnes-Jewish Hospital, 216 S. Kingshighway Blvd., Mailstop: 90-52-411, St. Louis, MO 63110; Telephone: 314-362-5407; Fax: 314-362-5407; E-mail: end0164@bjc.org

Additional Supporting Information may be found in the online version of this article.

Received: July 9, 2015; Revised: November 28, 2015; Accepted: December 17, 2015

2016 Society of Hospital Medicine DOI 10.1002/jhm.2545

Published online in Wiley Online Library (Wileyonlinelibrary.com).
}

native dosing strategy to the standard $10 \mathrm{U}$ of IV insulin traditionally used for this purpose. Beginning November 10, 2013, our computer prescriber order entry (CPOE) system automatically prepopulated a dose of $0.1 \mathrm{U} / \mathrm{kg}$ of body weight for any patients weighing $<95 \mathrm{~kg}$ (doses rounded to the nearest whole unit) when the acute hyperkalemia order set was utilized. The maximum dose allowed continued to be 10 U. The revised order set also changed nursing orders to require BG monitoring as frequently as every hour following the administration of insulin and dextrose for the treatment of hyperkalemia.

The purpose of this study is to investigate whether weight-based insulin dosing $(0.1 \mathrm{U} / \mathrm{kg})$ for patients weighing $<95 \mathrm{~kg}$, rather than a standard $10-\mathrm{U}$ insulin dose, resulted in fewer hypoglycemic episodes and patients affected. Secondarily, this study sought to determine the impact of weight-based insulin dosing on potassium-lowering effects of therapy and to detect any risk factors for development of hypoglycemia among this patient population.

\section{METHODS}

This institutional review board-approved, singlecenter, retrospective chart review examined patients for whom the physician order entry set for hyperkalemia therapy was utilized, including patients who weighed less than $95 \mathrm{~kg}$ and received regular insulin via weight-based dosing $(0.1 \mathrm{U} / \mathrm{kg}$ of body weight up to a maximum of $10 \mathrm{U}$ ) during the period November 10, 2013 to May 31, 2014, versus those who received fixed insulin dosing (10 $\mathrm{U}$ regardless of body weight) during the period May 1, 2013 to November 9, 2013. During each of these periods, the CPOE system autopopulated the recommended insulin dose, with the possibility for physician manual dose entry. Data 


\section{TABLE 1. Baseline Characteristics}

\begin{tabular}{lccc}
\hline Characteristics & $\begin{array}{c}10 \mathrm{U} \text { Insulin, } \\
\mathrm{n}=66\end{array}$ & $\begin{array}{c}0.1 \mathrm{U} / \mathrm{kg} \text { Insulin, } \\
\mathrm{n}=66\end{array}$ & $\begin{array}{c}P \text { Value } \\
(2-S i d e d)\end{array}$ \\
\hline Weight, $\mathrm{kg}$ & $69.9(14.2)$ & $74.2(12.6)$ & 0.07 \\
Age, $\mathrm{y}$ & $55.7(15.7)$ & $61.9(17.6)$ & 0.36 \\
Male gender & $37(56.1 \%)$ & $41(62.1 \%)$ & 0.60 \\
Caucasian race & $40(60.6 \%)$ & $37(56.1 \%)$ & 0.55 \\
Serum creatinine, mg/dL & $3.16(4.38)$ & $3.04(4.61)$ & 0.9 \\
Creatinine clearance $<30 \mathrm{~mL} /$ min & $41(62.1 \%)$ & $41(62.1 \%)$ & 0.6 \\
Dialysis & $20(30.3 \%)$ & $16(24.2 \%)$ & 0.56 \\
Baseline blood glucose, mg/dL & $166.0(71.7)$ & $147.3(48.0)$ & 0.08 \\
Received other insulin within 24 hours & $30(45.4 \%)$ & $25(37.9 \%)$ & 0.48 \\
$\quad$ of hyperkalemia treatment & & & \\
Received K+ supplement within 24 hours & $9(13.6 \%)$ & $11(16.7 \%)$ & 0.81 \\
$\quad$ of hyperkalemia treatment & & & \\
Baseline serum K+, mmo/L & $6.1(0.5)$ & $6.1(0.7)$ & 0.76 \\
Baseline serum K+ >6.0 mmol/L & $41(62.1 \%)$ & $33(50 \%)$ & 0.22 \\
No. of additional treatments for hyperkalemia & $1.5(0.8)$ & $1.4(0.9)$ & 0.49 \\
$\quad$ in addition to insulin/dextrose & & & \\
\end{tabular}

NOTE: Values are expressed as mean (standard deviation) or number (\%). collection was limited to the first use of insulin for hyperkalemia treatment per patient in each period.

Patients weighing $<95 \mathrm{~kg}$ were the focus of this study because they received $<10 \mathrm{U}$ of insulin under the weight-based dosing strategy. Patients were excluded from the study if they had a body weight $>95 \mathrm{~kg}$ or no weight recorded, were not administered insulin as ordered, received greater than the CPOEspecified insulin dose, or had no BG readings recorded within 24 hours of insulin administration. The first 66 patients within each group meeting all inclusion and exclusion criteria were randomly selected for analysis. This recruitment target was developed to provide enough patients for a meaningful analysis of hypoglycemia events based on previous reports from our institution. ${ }^{5}$

Hypoglycemia was defined as a recorded BG level $<70 \mathrm{mg} / \mathrm{dL}$ within 24 hours after insulin administration; severe hypoglycemia was defined as a recorded BG $<40 \mathrm{mg} / \mathrm{dL}$ within 24 hours. Individual episodes of hypoglycemia and severe hypoglycemia were recorded for each instance of such event separated by at least 1 hour from the time of the first recorded event. In addition, episodes of hypoglycemia or severe hypoglycemia and number of patients affected were assessed at within 6 hours, 6 to 12 hours, and 12 to 24 hours after insulin administration as separate subsets for statistical analysis.

For the purpose of assessing the potassium-lowering efficacy of weight-based versus traditional dosing of insulin, maximum serum potassium levels were examined in the 12-hour interval before the hyperkalemia order set was implemented and compared with minimum potassium levels in the 12 hours after insulin was administered. A comparison of the treatment groups assessed differences between the mean decrease
TABLE 2. Hypoglycemia Outcomes and Impact on Potassium Values

\begin{tabular}{lccc}
\hline Outcomes & $\begin{array}{c}10 \mathrm{U} \text { Insulin, } \\
\mathrm{n}=66\end{array}$ & $\begin{array}{c}0.1 \mathrm{U} / \mathrm{kg} \text { Insulin, } \\
\mathrm{n}=66\end{array}$ & $\begin{array}{c}P \text { Value } \\
\text { (2-Sided) }\end{array}$ \\
\hline Hypoglycemia, $<70 \mathrm{mg} / \mathrm{dL}$ & & & \\
$\quad$ No. of patients & $13(19.7 \%)$ & $7(10.6 \%)$ & 0.22 \\
No. of events total & $18(27.3 \%)$ & $8(12.1 \%)$ & 0.05 \\
$\quad$ No. of events 0-6 hours & $7(10.6 \%)$ & $3(4.5 \%)$ & 0.32 \\
No. of events 6-12 hours & $5(7.6 \%)$ & $2(3.0 \%)$ & 0.44 \\
No. of events 12-24 hours & $6(9.1 \%)$ & $3(4.5 \%)$ & 0.49 \\
Severe hypoglycemia & & & \\
$\quad$ No. of patients & $2(3.0 \%)$ & $1(1.5 \%)$ & $>0.99$ \\
No. of events total & $2(3 \%)$ & $1(1.5 \%)$ & $>0.99$ \\
Potassium-lowering effects & & & \\
Minimum K+ after therapy, mmol/L (SD) & $4.9(0.7)$ & $4.8(0.7)$ & 0.84 \\
Minimum serum K+ <5.0 mmol/L (\%) & $37(56.1 \%)$ & $35(53.0 \%)$ & 0.32 \\
Average K+ decrease, mmol/L (SD) & $1.35(0.97)$ & $1.34(0.94)$ & 0.94 \\
Repeat treatment given (\%) & $24(36.4 \%)$ & $24(36.4 \%)$ & $>0.99$ \\
\hline
\end{tabular}

NOTE: Values are expressed as number (\%) unless indicated otherwise. Abbreviations: SD, standard deviation.

in serum potassium from baseline, the mean minimum potassium achieved, the number of patients achieving minimum potassium below $5.0 \mathrm{mEq} / \mathrm{L}$, and the number of patients who subsequently received repeat treatment for hyperkalemia within 24 hours of treatment with insulin.

Statistical analysis was conducted utilizing $\chi^{2}$ and Fisher exact tests for nominal data and Student $t$ test for continuous data to detect statistically significant differences between the groups. Binomial logistic multivariable analysis using a backward stepwise approach was used to determine factors for development of hypoglycemia, analyzed on a per-patient basis to prevent characteristics from being over-represented when events occurred multiple times to a single patient. All analyses were completed by using SPSS version 18 (SPSS Inc., Chicago, IL).

\section{RESULTS}

In total, 1734 entries were available for the acute hyperkalemia order set with insulin during the 2 periods investigated. Only 464 patients were eligible for manual chart review once weight-based exclusions were identified by electronic database, with additional exclusion criteria later extracted from patient charts. Patients in both treatment groups were fairly well balanced, with a slightly lower body weight in the 10-U insulin group recorded (Table 1). Patients in the weight-based dosing group received between 4 and 9 $\mathrm{U}$ of insulin, depending on body weight.

A reduction in the number of hypoglycemic episodes was detected in the weight-based dosing group of $56 \%$ within 24 hours, from 18 to 8 events ( $P=$ 0.05 ) (Table 2). The number of hypoglycemic events in every subset of time intervals was likewise reduced by at least $50 \%$ using weight-based dosing (from 7 to 3 events within 6 hours, from 5 to 2 events in 6-12 hours, from 6 to 3 events in 12-24 hours). The 
number of patients who experienced hypoglycemia within 24 hours after receiving insulin also was reduced in the weight-based dosing group by $46 \%(P$ $=0.22$ ).

Potassium lowering was comparable across both dosing strategies in every measure assessed (Table 2). Multivariate analysis revealed that baseline BG $<140$ $\mathrm{mg} / \mathrm{dL}$ (adjusted odds ratio: 4.3, 95\% confidence interval $[\mathrm{CI}]: 1.4-13.7, P=0.01$ ) and female gender (adjusted odds ratio: $3.2,95 \%$ CI: 1.1-9.1, $P=0.03$ ) were associated with an increased risk of hypoglycemia. Other factors, including administration of insulin beyond that for hyperkalemia treatment and use of additional hypoglycemic agents, were not associated with the development of hypoglycemia, which is consistent with previous reports. ${ }^{6}$

\section{CONCLUSIONS}

Our findings indicate that using a weight-based approach to insulin dosing when treating hyperkalemia may lead to a reduction in hypoglycemia without sacrificing the efficacy of potassium lowering. Females and patients with glucose values $<140 \mathrm{mg} / \mathrm{dL}$ were at increased risk of hypoglycemia in this cohort. Based on the results of this research, a weight-based dosing strategy of $0.1 \mathrm{U} / \mathrm{kg}$ IV insulin up to a maximum of $10 \mathrm{U}$ should be considered, with further research desirable to validate these results.

This study was strengthened by the inclusion of all patients regardless of baseline glucose, baseline potassium, administration of other insulins, level of renal impairment, or symptomatic display of hypoglycemia or cardiac dysfunction, thus providing a broad representation of patients treated for acute hyperkalemia. This pilot study was limited in its scope by data collection for only 66 randomized patients per group rather than the entire patient population. In addition, the study utilized patient information from a single site, with few ethnicities represented. Validation of this research using a larger sample size should include greater variation in the patients served. Our inclusion of a hypoglycemia definition up to 24 hours after treatment may also be criticized. However, this is similar to previous reports and allows for a liberal time period for follow-up glucose monitoring to be recorded. ${ }^{7}$

Because of its small sample size and the low event rate, this study was unable to draw conclusions about the ability of weight-based insulin dosing to affect severe hypoglycemic events $(<40 \mathrm{mg} / \mathrm{dL})$. A study of more than 400 patients would be necessary to find statistically significant differences in the risk of severe hypoglycemia. Furthermore, because we did not examine the results from all patients in this cohort, we cannot conclusively determine the impact of treatment. The retrospective nature of this study limited our ability to capture hypoglycemic episodes during periods in which BG levels were not recorded. Additionally, changes to the post-treatment glucose monitoring protocol may have also affected the incidence of hypoglycemia in 2 potential ways. First, early and unrecorded interventions may have occurred in patients with a trend toward hypoglycemia. Second, the longer time to follow-up in the non-weight-based group may have led to additional hypoglycemic episodes being missed. A prospective trial design could provide more comprehensive information about patient response to weight-based versus traditional dosing of IV insulin for hyperkalemia. Further investigations on reducing adverse effects of insulin when treating hyperkalemia should focus on female patients and those with lower baseline BG values. Additionally, as newer agents to treat hyperkalemia are developed and tested, the approach to management should be revisited. ${ }^{8-10}$

Disclosures: Garry S. Tobin, MD, lectures or is on the speakers bureau for Eli Lilly, Jansen, Boehringher Ingelheim, and Novo Nordisk, and performs data safety monitoring for Novo Nordisk. The authors report no other potential conflicts of interest.

\section{References}

1. Acker CG, Johnson JP, Palvelsky P, Greenberg A. Hyperkalemia in hospitalized patients: causes, adequacy of treatment, and results of an attempt to improve physician compliance with published therapy guidelines. Arch Intern Med. 1998;158:917-924.

2. 2010 American Heart Association guidelines for cardiopulmonary resuscitation and emergency cardiovascular care. Part 12.6: cardiac arrest associated with life-threatening electrolyte disturbances. Circulation. 2010;122:S829-S861.

3. Centers for Medicare \& Medicaid Services. NQF 2362 and NQF 2363. Available at: https://www.cms.gov/Medicare/Quality-InitiativesPatient-Assessment-Instruments/QualityMeasures/index.html? redirect $=$ /QUALITYMEASURES/. Accessed March 6, 2015.

4. Brodovicz KG, Mehta V, Zhang Q, et al. Association between hypoglycemia and inpatient mortality and length of hospital stay in hospitalized, insulin-treated patients. Curr Med Res Opin. 2013; 29(2):101-107.

5. Schafers S, Naunheim R, Vijayan A, Tobin G. Incidence of hypoglycemia following insulin-based acute stabilization of hyperkalemia treatment. J Hosp Med. 2012;7(3):239-242.

6. Apel J, Reutrakul S, Baldwin D. Hypoglycemia in the treatment of hyperkalemia with insulin in patients with end-stage renal disease. Clin Kidney J. 2014;7(3):248-250.

7. Elliott MB, Schafers S, McGill J, Tobin G. Prediction and prevention of treatment-related inpatient hypoglycemia. J Diabetes Sci Technol. 2012;6(2):302-309

8. Kosiborod M, Rasmussen HS, Lavin P, et al. Effect of sodium zirconium cyclosilicate on potassium lowering for 28 days among outpatients with hyperkalemia: the HARMONIZE randomized clinical trial. JAMA. 2014;312(21):2223-2233.

9. Packham DK, Rasmussen HS, Lavin PT, et al. Zirconium cyclosilicate in hyperkalemia. N Engl J Med. 2015;372:222-231.

10. Weir MR, Bakris GL, Bushinsky DA, et al. Patiromer in patients with kidney disease and hyperkalemia receiving RAAS inhibitors. $N$ Engl J Med. 2015;372:211-221. 